\title{
NEW EVOLUTIONARY SEQUENCES FOR HOT H-DEFICIENT WHITE DWARFS ON THE BASIS OF A FULL ACCOUNT OF PROGENITOR EVOLUTION
}

\author{
L. G. Althaus ${ }^{1,2}$, J. A. Panei ${ }^{1,3}$, M. M. Miller Bertolami ${ }^{1,3,4}$, E. García-Berro ${ }^{2,5}$, A. H. Córsico ${ }^{1,3}$, A. D. Romero ${ }^{1,3}$, \\ S. O. KEPLER ${ }^{6}$, AND R. D. ROHRMANN ${ }^{7}$ \\ ${ }^{1}$ Facultad de Ciencias Astronómicas y Geofísicas, Universidad Nacional de La Plata, Paseo del Bosque s/n, (1900) La Plata, Argentina; althaus@fcaglp.unlp.edu.ar \\ ${ }^{2}$ Departament de Física Aplicada, Universitat Politècnica de Catalunya, c/Esteve Terrades 5, 08860 Castelldefels, Spain \\ ${ }^{3}$ Instituto de Astrofísica de La Plata, IALP (CCT La Plata), CONICET-UNLP, Argentina \\ ${ }^{4}$ Max Planck Institut für Astrophysik, Karl-Schwarzschild-Str. 1, 85748 Garching, Germany \\ ${ }^{5}$ Institute for Space Studies of Catalonia, c/Gran Capità 2-4, Edif. Nexus 104, 08034 Barcelona, Spain \\ ${ }^{6}$ Instituto de Física, Universidade Federal do Rio Grande do Sul, 91501-970 Porto Alegre, RS, Brazil \\ ${ }^{7}$ Observatorio Astronómico, Universidad Nacional de Córdoba, Laprida 854, (5000) Córdoba, Argentina \\ Received 2009 July 13; accepted 2009 September 16; published 2009 October 2
}

\begin{abstract}
We present full evolutionary calculations appropriate for the study of hot hydrogen-deficient DO white dwarfs, PG 1159 stars, and DB white dwarfs. White dwarf sequences are computed for a wide range of stellar masses and helium envelopes on the basis of a complete treatment of the evolutionary history of progenitors stars, including the core hydrogen and helium burning phases, the thermally pulsing asymptotic giant branch phase, and the born-again episode that is responsible for the hydrogen deficiency. We also provide colors and magnitudes for the new sequences for $T_{\text {eff }}<40,000 \mathrm{~K}$, where the NLTE effects are not dominant. These new calculations provide a homogeneous set of evolutionary tracks appropriate for mass and age determinations for both PG 1159 stars and DO white dwarfs. The calculations are extended down to an effective temperature of $7000 \mathrm{~K}$. We applied these new tracks to redetermine stellar masses and ages of all known DO white dwarfs with spectroscopically determined effective temperatures and gravities, and compare them with previous results. We also compare for the first time consistent mass determinations for both DO and PG 1159 stars, and find a considerably higher mean mass for the DO white dwarfs. We discuss as well the chemical profile expected in the envelope of variable DB white dwarfs from the consideration of the evolutionary history of progenitor stars. Finally, we present tentative evidence for a different evolutionary channel, other than that involving the PG 1159 stars, for the formation of hot, hydrogen-deficient white dwarfs.
\end{abstract}

Key words: stars: abundances - stars: AGB and post-ABG - stars: evolution - stars: interiors - stars: variables: other - white dwarfs

Online-only material: color figures

\section{INTRODUCTION}

White dwarf stars are the end product of the evolution of the vast majority of stars. Indeed, more than $97 \%$ of all stars in our Galaxy are expected to end their lives as white dwarfs. For this reason, the present population of white dwarfs contains valuable information about the evolution of individual stars, the previous history of the Galactic populations (Isern et al. 1998; Torres et al. 2002), and the rate of star formation (Díaz-Pinto et al. 1994). In addition, white dwarfs have potential applications as reliable cosmic clocks to infer the age of a wide variety of stellar populations (Winget et al. 1987; García-Berro et al. 1988; Hansen et al. 2007), and to place constraints on elementary particles (Córsico et al. 2001; Isern et al. 2008). Recent reviews on the properties and evolution of white dwarfs and of their applications are those of Winget \& Kepler (2008) and Fontaine \& Brassard (2008).

Traditionally, white dwarfs have been classified into two distinct families according to the main constituent of their surface: those with a hydrogen $(\mathrm{H})$-dominated atmospherethe DA spectral type-and those with a helium (He)-rich surface composition - the non-DA white dwarfs. The latter, that comprise about $\sim 20 \%$ of known white dwarfs, are usually divided into three subclasses: the DO spectral type (with effective temperatures $45,000 \mathrm{~K} \leqslant T_{\text {eff }} \leqslant 200,000 \mathrm{~K}$ ) that shows relatively strong lines of singly ionized $\mathrm{He}$ (He II); the
DB type $\left(11,000 \mathrm{~K} \leqslant T_{\text {eff }} \leqslant 30,000 \mathrm{~K}\right)$, with strong neutral He (He I) lines; and the DC, DQ, and DZ types $\left(T_{\text {eff }}<11,000 \mathrm{~K}\right)$ showing traces of carbon and metals in their spectra. As a DO white dwarf evolves, the He II recombines to form He I, ultimately transforming into a DB white dwarf. The transition from DO to the cooler DB stage is interrupted by the non-DA gap (that occurs at 30,000 K $<T_{\text {eff }}<45,000 \mathrm{~K}$ ) where few objects with $\mathrm{H}$-deficient atmospheres have been observed (Eisenstein et al. 2006). To this list, we have to add the discovery of a new white dwarf spectral type with carbon-dominated atmospheres, the "hot DQ" white dwarfs, with $T_{\text {eff }} \sim 20,000 \mathrm{~K}$ (Dufour et al. 2007, 2008).

DO white dwarfs are usually thought to be the progeny of PG 1159 stars (Dreizler \& Werner 1996; Unglaub \& Bues 2000; Althaus et al. 2005), hot stars with H-deficient and He-, C- and O-rich surface layers (Werner \& Herwig 2006). A large fraction of PG 1159 stars is believed to be the result of a born-again episode, i.e., a very late thermal pulse (VLTP) experienced by a white dwarf during its early cooling phase (Fujimoto 1977; Schönberner 1979; Iben et al. 1983; Althaus et al. 2005). During the VLTP, most of the $\mathrm{H}$ content of the remnant is violently burned (Herwig et al. 1999; Miller Bertolami et al. 2006). As a result, the remnant is forced to evolve rapidly back to the asymptotic giant branch (AGB) and finally into the central star of a planetary nebula as a hot H-deficient object and with a surface composition resembling that of the intershell region 
chemistry of AGB star models - typical mass abundances are $X_{\mathrm{He}} \simeq 0.33, X_{\mathrm{C}} \simeq 0.5$, and $X_{\mathrm{O}} \simeq 0.17$, though notable variations are found from star to star (Dreizler \& Heber 1998; Werner 2001). Eventually, gravitational settling acting during the early stages of white dwarf evolution causes He to float and heavier elements to sink down, giving rise to an He-dominated surface, and turning the PG 1159 star into a DO white dwarf (Unglaub \& Bues 2000). During this stage, the evolution of the star is dictated essentially by neutrino losses and the release of gravothermal energy (O'Brien \& Kawaler 2000; Althaus et al. 2005). Because these white dwarfs are the hottest ones, the evolution during the DO stage proceeds very fast, with typical evolutionary timescales $\sim 1$ Myr.

Several works have been devoted to the identification and spectral analysis of H-deficient objects like PG 1159 and DO white dwarfs - see Dreizler \& Werner (1996), Dreizler et al. (1997), and references therein for earlier studies of spectroscopically confirmed DO white dwarfs. Particularly relevant is the star KPD0005+5106, the hottest known DO white dwarf with an effective temperature of about 200,000 K (Werner et al. 2008a), much higher than previously thought (Dreizler $\&$ Werner 1996). With the advent of large surveys, like the Sloan Digital Sky Survey (SDSS; York et al. 2000) the number of spectroscopically identified H-deficient stars has increased considerably. For instance, Krzesiński et al. (2004) reported 15 spectroscopically identified DO stars-13 of which were new discoveries-from the SDSS Data Release 1 (Abazajian et al. 2003), which contains $\approx 2500$ white dwarfs (Kleinman et al. 2004). Also, Eisenstein et al. (2006) reported 31 DO white dwarfs and 10 PG 1159 stars, from their catalog based on SDSS DR4 (Adelman-McCarthy et al. 2006). Hügelmeyer et al. (2005) performed spectral analyses of 16 hot H-deficient stars from DR1, DR2, and DR3 catalogs, where nine were classified as DO and five as PG 1159 stars. More recently, Hügelmeyer et al. (2006) extended this work to 13 DO and 4 PG 1159 stars-see also Werner et al. (2004). These authors derived effective temperatures, surface gravities, and stellar masses for their sample, and for that of Hügelmeyer et al. (2005), and discussed the evolutionary connection between DO white dwarfs and PG 1159 stars.

To infer the mass and age of observed DO white dwarfs, evolutionary calculations for these stars are required. However, most of the existing calculations that are employed in the determination of evolutionary parameters of non-DA white dwarf stars (Wood 1995; Benvenuto \& Althaus 1999) are not entirely consistent with the evolutionary history that leads to the formation of H-deficient stars. Even though significant deviations are not expected at low effective temperatures, this lack of consistency becomes relevant for the high effective temperatures characteristic of hot DO white dwarfs. In addition, the lack of evolutionary calculations covering the whole stage from the domain of luminous PG 1159 to the hot white dwarf stage prevents from a consistent mass determination for the DOs and PG 1159 stars (Dreizler \& Werner 1996). To our knowledge, the only exception is the study of Althaus et al. (2005), who computed the formation and evolution of a $0.588 M_{\odot}$ white dwarf remnant from the zero-age main sequence (ZAMS) through the born-again and PG 1159 stages to the white dwarf regime. This paper is precisely intended to fill this gap, by computing new evolutionary tracks and mass-radius relations appropriate for both non-DA white dwarfs and PG 1159 stars. Sequences are derived from a full and self-consistent treatment of the complete evolutionary history of progenitor stars with different masses evolved through the born-again episode. In this way, the white dwarf evolutionary calculations presented here are not affected by inconsistencies arising from artificial procedures to generate starting white dwarf configurations. In addition, the computation of the evolutionary history of progenitor stars gives us the amount of He left in the white dwarf as well as the chemical profiles expected not only for the carbon-oxygen core, but also for the partially degenerate regions above the core, of relevance for the white dwarf cooling phase.

The calculations presented here constitute a homogeneous set of evolutionary tracks aimed to study both PG 1159 and DO white dwarf stars, as well as the DB white dwarfs. The paper is organized as follows. In Section 2, we describe the main physical inputs to the models. We also describe the main characteristics of the initial models and some details of the evolutionary computations. In Section 3, we present the results for DO white dwarf evolutionary sequences. We apply the sequences to redetermine stellar masses and ages of observed DO white dwarfs, and we derive mass-radius relations. In addition, we show the results for the case of DO white dwarf sequences with very thin He-rich envelopes. We close the paper in Section 4 by discussing and summarizing the implications of our results, particularly regarding the origin of the observed DO white dwarfs.

\section{COMPUTATIONAL DETAILS}

\subsection{Input Physics}

The evolutionary calculations presented in this work were done with the LPCODE stellar evolutionary code we employed in our previous studies on the formation of the H-deficient stars through late He flashes, like PG 1159 and extreme horizontal branch stars (Althaus et al. 2005; Miller Bertolami \& Althaus 2006; Miller Bertolami et al. 2008). More recently, the code was used to compute the formation of hot DQ white dwarfs (Althaus et al. 2009b) as well as the formation and evolution of He-core white dwarfs with high metallicity progenitors (Althaus et al. 2009c). Details of LPCODE can be found in these works. In particular, the code considers a simultaneous treatment of non-instantaneous mixing and burning of elements, which is of primary importance for the calculation of chemical abundance changes during the short-lived evolutionary stages characteristic of unstable burning episodes, like the bornagain stage, from which our starting $\mathrm{H}$-deficient white dwarf configurations are derived, see Section 2.1. Nuclear reaction rates are from Caughlan \& Fowler (1988) and Angulo et al. (1999), and are detailed in Althaus et al. (2005). In particular, the ${ }^{12} \mathrm{C}(\alpha, \gamma){ }^{16} \mathrm{O}$ reaction rate was taken from Angulo et al. (1999), which is about twice as large as that of Caughlan \& Fowler (1988). A moderate, diffusive overshooting in the core and in the envelope is allowed during pre-white-dwarf evolution.

For the white dwarf regime, we considered the following main physical ingredients. Neutrino emission rates for pair, photo, and bremsstrahlung processes are those of Itoh et al. (1996). For plasma processes, we included the treatment presented in Haft et al. (1994). Radiative opacities are those of the OPAL project (Iglesias \& Rogers 1996), including carbon- and oxygen-rich composition. We adopted the conductive opacities of Cassisi et al. (2007). This prescription covers the whole regime where electron conduction is relevant. For the highdensity regime, we used the equation of state of Segretain et al. 
Table 1

Initial and Final Stellar Mass (in Solar Units), and the Total Mass of $\mathrm{He}$ Content Left in the White Dwarf for the Evolutionary Sequences Considered in this Work

\begin{tabular}{lcc}
\hline \hline$M_{\mathrm{WD}}$ & $M_{\text {ZAMS }}$ & $M_{\mathrm{He}}$ \\
\hline 0.515 & 1.00 & 0.0219 \\
0.530 & 1.00 & 0.0090 \\
0.542 & 1.00 & 0.0072 \\
0.565 & 2.20 & 0.0067 \\
0.584 & 2.50 & 0.0060 \\
0.609 & 3.05 & 0.0058 \\
0.664 & 3.50 & 0.0036 \\
0.741 & 3.75 & 0.0019 \\
0.870 & 5.50 & 0.0009 \\
\hline
\end{tabular}

(1994), which accounts for all the important contributions for both the liquid and solid phases-see Althaus et al. (2007b) and references therein. For the low-density regime, we used an updated version of the equation of state of Magni \& Mazzitelli (1979). The release of latent heat upon crystallization was also considered. In particular, for our calculations, crystallization sets in when the ion coupling constant reaches $\Gamma=180$, where $\Gamma \equiv\left\langle Z^{5 / 3}\right\rangle e^{2} / a_{e} k_{\mathrm{B}} T$ and $a_{e}$ is the interelectronic distance. We considered a latent heat release of $k_{\mathrm{B}} T$ per ion, which was spread over the range $175<\Gamma<185$. Convection was treated in the formalism of the mixing-length theory as given by the ML2 parameterization (Tassoul et al. 1990).

All our white dwarf sequences were computed in a consistent way with the evolution of the chemical abundance distribution caused by element diffusion along the whole cooling phase. In particular, we considered gravitational settling and chemical diffusion of ${ }^{4} \mathrm{He},{ }^{12} \mathrm{C},{ }^{13} \mathrm{C},{ }^{14} \mathrm{~N}$, and ${ }^{16} \mathrm{O}$ - see Althaus et al. (2003) for details. In our simulations, we obtain high amounts of ${ }^{13} \mathrm{C}$ and ${ }^{14} \mathrm{~N}$ at the VLTP, in agreement with observational inferences in the Sakurai object and, in the case of ${ }^{14} \mathrm{~N}$, in some PG 1159 stars (Werner \& Herwig 2006). Our treatment of time-dependent diffusion is based on the multicomponent gas treatment presented in Burgers (1969). Diffusion velocities are evaluated at each evolutionary time step. For the white dwarf evolution, is worth mentioning that opacity is calculated taking into account the diffusion predictions for the heavy element composition.

\subsection{Initial Models}

The initial models for our white dwarf sequences correspond to realistic PG 1159 stellar configurations derived from the full evolutionary calculations of their progenitor stars for solar metallicity (Miller Bertolami \& Althaus 2006). All the sequences were computed from the ZAMS through the thermally pulsing and mass-loss phases on the AGB and finally to the bornagain stage where the remaining $\mathrm{H}$ is violently burnt. After the born-again episode, the $\mathrm{H}$-deficient, quiescent He-burning remnants evolve at constant luminosity to the domain of PG 1159 stars with a surface chemical composition rich in $\mathrm{He}$, carbon, and oxygen (Miller Bertolami \& Althaus 2006). This new generation of PG 1159 evolutionary models has succeeded in explaining both the spread in surface chemical composition observed in most PG 1159 stars and the location of the GW Vir instability strip in the $\log T_{\text {eff }}-\log g$ plane (Córsico et al. 2006), as well as the short born-again timescales of V4334 Sgr (Miller Bertolami et al. 2006). To our knowledge, this is the first time that such a detailed treatment of the evolutionary history of progenitors stars with different initial stellar masses is taken into account

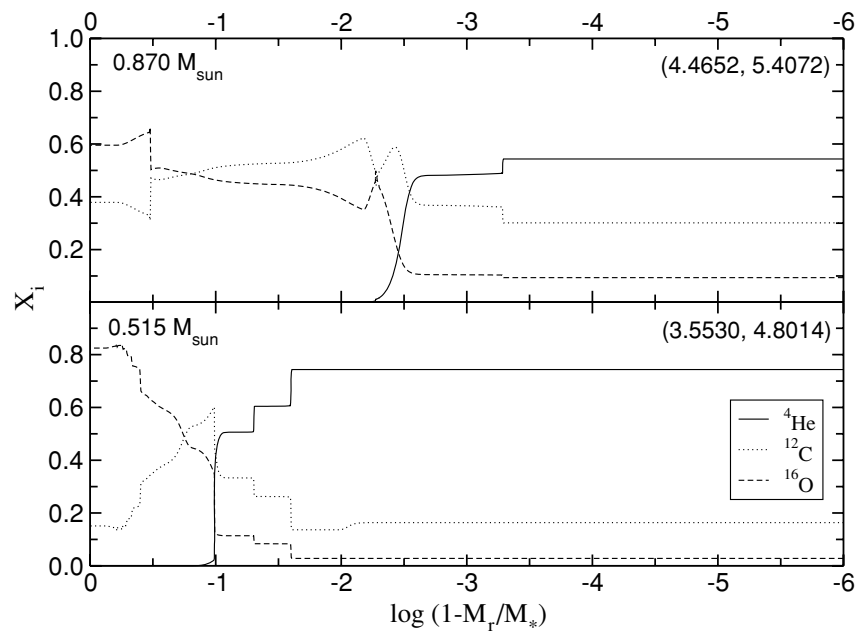

Figure 1. Abundance by mass of ${ }^{4} \mathrm{He},{ }^{12} \mathrm{C}$, and ${ }^{16} \mathrm{O}$ as a function of the outer mass fraction $\log \left(1-M_{r} / M_{*}\right)$ for PG 1159 models corresponding to the sequences of 0.870 (top panel) and $0.515 M_{\odot}$ (bottom panel). Luminosity and effective temperature $\left(\log \left(L / L_{\odot}\right) ; \log T_{\text {eff }}\right)$ are specified for each model.

in the calculation of cooling sequences for $\mathrm{H}$-deficient white dwarfs.

Specifically, we computed nine H-deficient white dwarf evolutionary sequences. In Table 1 , we list the stellar masses of the resulting white dwarfs, together with the initial masses of the progenitor stars at the ZAMS. Also listed in Table 1 is the mass of He left by previous evolution. Note that for these sequences, which cover most of the observed stellar mass range of H-deficient white dwarfs, the expected range of He envelope masses spans more than 1 order of magnitude. Due to He burning during the PG 1159 stage, the mass of He left when the remnant reaches its cooling track is considerably lower than that obtained shortly after the born-again episode $\left(0.0036\right.$ and $0.0075 M_{\odot}$, respectively, for the $0.664 M_{\odot}$ sequence). For the $1 M_{\odot}$ model, two different sequences were computed with two different massloss rates during the AGB evolution. In this way, we obtain a different number of thermal pulses and, in the end, two different remnant masses of 0.530 and $0.542 M_{\odot}$, respectively. Finally, the sequence with $0.515 M_{\odot}$ was specifically computed to reproduce the observational features of low-mass, He-enriched PG 1159 stars, like MCT 0130-1937 and HS 1517+7403-see Miller Bertolami \& Althaus (2006) and Althaus et al. (2007a). This model was extracted from the full evolution of a $1 M_{\odot}$ model star that experiences its first thermal pulse as a late thermal pulse (LTP) after leaving the AGB. We adopt the massloss rates of Blöcker (1995) multiplied by a 0.2 factor. Mass loss during the departure from the AGB has been arbitrarily set to obtain a final helium shell flash during the early white dwarf cooling phase. Our calculations cover the hot pre-white-dwarf stages (PG 1159 regime) and the advanced phases of evolution down to an effective temperature of $7000 \mathrm{~K}$, therefore covering all the DB cooling sequence.

In Figure 1, we show the mass abundances of ${ }^{4} \mathrm{He},{ }^{12} \mathrm{C}$, and ${ }^{16} \mathrm{O}$ throughout the deep interior of two PG 1159 star models corresponding to the sequences with 0.515 and $0.870 M_{\odot}$. The models shown correspond to evolutionary stages at high luminosities where the chemical profile at the bottom of the Herich envelope is being modified by residual He shell burning. Note the dependence of the composition profile on the stellar mass, which emphasizes the need for a detailed knowledge of the progenitor history for a realistic treatment of white dwarf 
Table 2

Main Parameters Associated to the Model with Highest $T_{\text {eff }}$ for Each Sequence

\begin{tabular}{lcccccc}
\hline \hline$M / M_{\odot}$ & $\log T_{\text {eff }}(\mathrm{K})$ & $\log \left(L / L_{\odot}\right)$ & $\log g\left(\mathrm{~cm} \mathrm{~s}^{-2}\right)$ & $X_{\mathrm{He}}$ & $X_{\mathrm{C}}$ & $X_{\mathrm{O}}$ \\
\hline 0.515 & 5.0634 & 2.6868 & 6.6713 & 0.7437 & 0.1637 & 0.0279 \\
0.530 & 5.1366 & 2.9762 & 6.6876 & 0.3301 & 0.3944 & 0.1716 \\
0.542 & 5.1650 & 3.0546 & 6.7412 & 0.2805 & 0.4064 & 0.2127 \\
0.565 & 5.2105 & 3.1565 & 6.8304 & 0.3632 & 0.2737 & 0.2173 \\
0.584 & 5.2398 & 3.2574 & 6.8615 & 0.3947 & 0.3060 & 0.1704 \\
0.609 & 5.2819 & 3.3533 & 6.9518 & 0.4990 & 0.3465 & 0.1033 \\
0.664 & 5.3578 & 3.3611 & 7.0813 & 0.4707 & 0.3260 & 0.1234 \\
0.741 & 5.4535 & 3.8069 & 7.2701 & 0.4795 & 0.3361 & 0.1390 \\
0.870 & 5.5829 & 4.0961 & 7.5679 & 0.5433 & 0.3012 & 0.0938
\end{tabular}

Note. The tabulated abundances (by mass fraction) correspond to the surface value.

evolution. The chemical stratification of our H-deficient prewhite-dwarf models consists of a carbon-oxygen core, which emerges from core He burning in prior stages. The core chemical profile is typical of situations in which extra mixing episodes beyond the fully convective core during the core He burning, like moderate overshooting and/or semiconvection, are allowedsee Straniero et al. (2003) and also Prada Moroni \& Straniero (2007) for the consequences on white dwarf evolution. The core is surrounded by a homogeneous and extended envelope rich in $\mathrm{He}$, carbon, and oxygen, which is the result of prior mixing and burning events during the thermally pulsing AGB and born-again phases. The surface abundance of He of our pre-white-dwarf models is within 0.28 and 0.75 by mass (see Table 2), which is in agreement with the range of observed $\mathrm{He}$ abundances in most PG 1159 stars (Werner \& Herwig 2006). These abundances are not only determined by the stellar mass, but also by the number of thermal pulses during the AGB phase, by the inner penetration of the convective envelope, and by the mass lost after the complete burning of protons (Miller Bertolami \& Althaus 2006). Finally, we have removed any $\mathrm{H}$ from our PG 1159 models, despite that some $\mathrm{H}$ is expected to be present in PG 1159 stars. This is particularly true if some of these stars result from a LTP episode. Here, the $\mathrm{H}$ content with which the progenitor star departs from the AGB for the first time is not burnt during the late helium flash but instead diluted to deeper layers. In this case, the presence of H in PG 1159 stars may be responsible for the fact that not all PG 1159 stars evolve to DO white dwarfs, see Section 4. By contrast, during the VLTP, only traces of $\mathrm{H}$ survive the violent $\mathrm{H}$ burning, see Miller Bertolami et al. (2006). It is expected that a large fraction of this remaining $\mathrm{H}$ is removed by mass loss during the following return to the giant state and Sakurai state.

\section{EVOLUTIONARY RESULTS}

\subsection{Sequences with Thick Envelopes}

In Figure 2, we show the evolution of our $\mathrm{H}$-deficient sequences both in the Hertzsprung-Russell diagram and in the $\log T_{\text {eff }}-\log g$ plane. The evolutionary sequences shown cover from the PG 1159 stage at intermediate gravities to the white dwarf domain. Thus, these sequences constitute a homogeneous set of tracks that consistently cover both evolutionary stages. Some characteristics of them at the highest effective temperature are listed in Table 2.

During the PG 1159 and hot white dwarf stages, ${ }^{4} \mathrm{He},{ }^{12} \mathrm{C}$, and ${ }^{16} \mathrm{O}$ are by far the dominant species in the envelope of our models. Among the main remaining constituents are ${ }^{13} \mathrm{C},{ }^{14} \mathrm{~N}$,

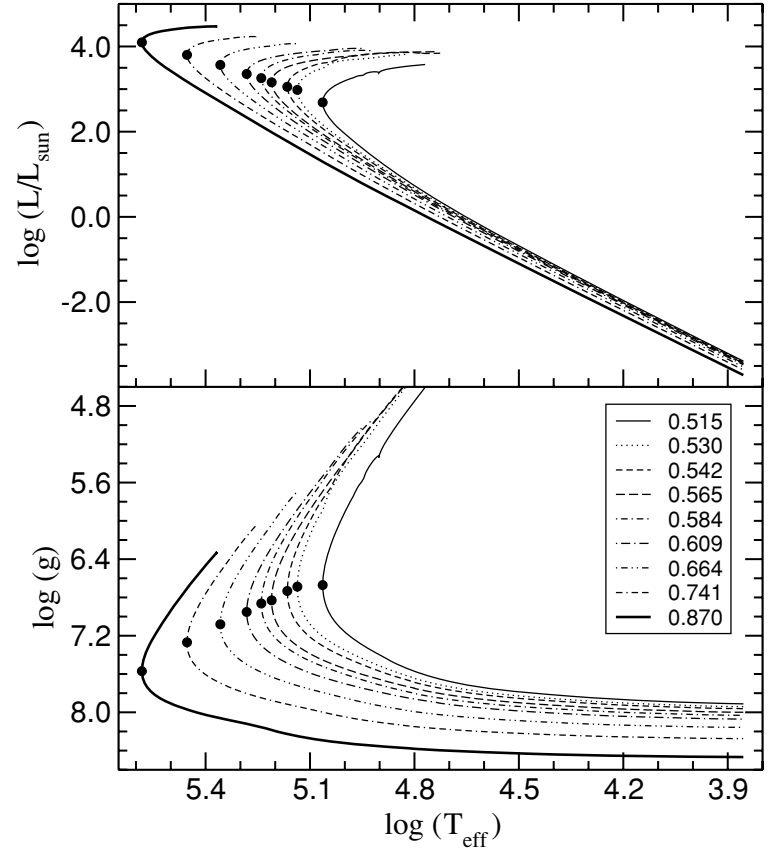

Figure 2. Upper panel: Hertzsprung-Russell diagram for our evolutionary sequences. Bottom panel: evolution in the $\log T_{\text {eff }}-\log g$ plane. Filled dots on each track indicate the instant with the highest effective temperature, which we adopt as our time origin. Mass values are given in solar units.

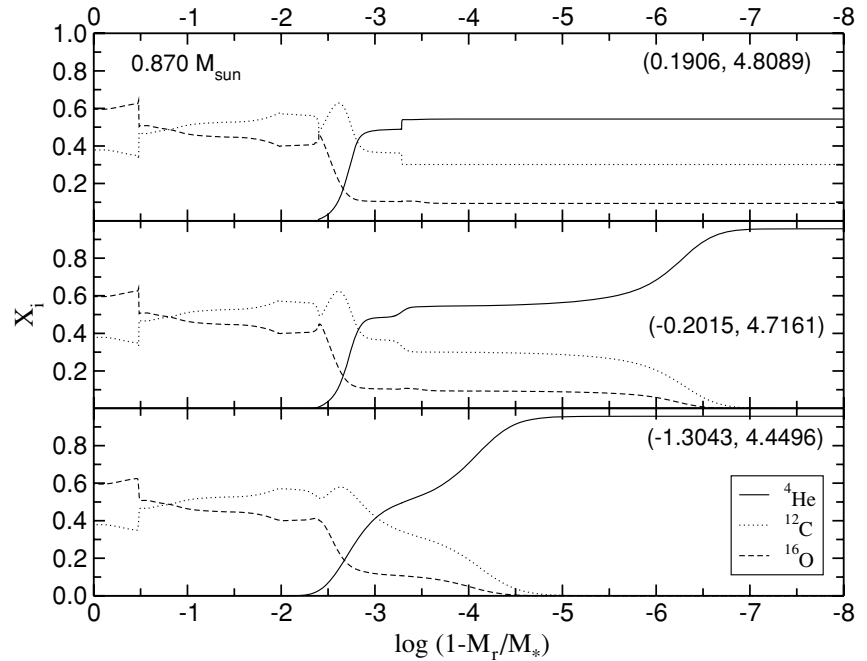

Figure 3. Internal abundance distribution of ${ }^{4} \mathrm{He},{ }^{12} \mathrm{C}$, and ${ }^{16} \mathrm{O}$ as a function of the outer mass fraction $\log \left(1-M_{r} / M_{*}\right)$ for our $0.870 M_{\odot}$ sequence at various selected stages on its cooling branch, as specified by the luminosity and effective temperature values $\left(\log \left(L / L_{\odot}\right), \log T_{\text {eff }}\right)$ in each panel.

and ${ }^{22} \mathrm{Ne}$. In particular, as a result of the violent $\mathrm{H}$ burning during the born-again episode, ${ }^{13} \mathrm{C}$ reaches abundances as high as 0.05 by mass in the envelope. Once He burning becomes virtually extinct after the point of maximum effective temperature, the H-deficient remnants settle upon their cooling track. Here, the chemical abundance distribution will be strongly modified by the various diffusion processes acting during white dwarf evolution. A glimpse of the inner chemistry variations that occur during this stage is given in Figures 3 and 4 for the 0.870 and $0.515 M_{\odot}$ sequences, respectively. The upper panel of each figure shows the chemical stratification at the start of the cooling track. Clearly, gravitational settling causes He to float to the surface and heavier elements to sink. Thus, as the evolution 


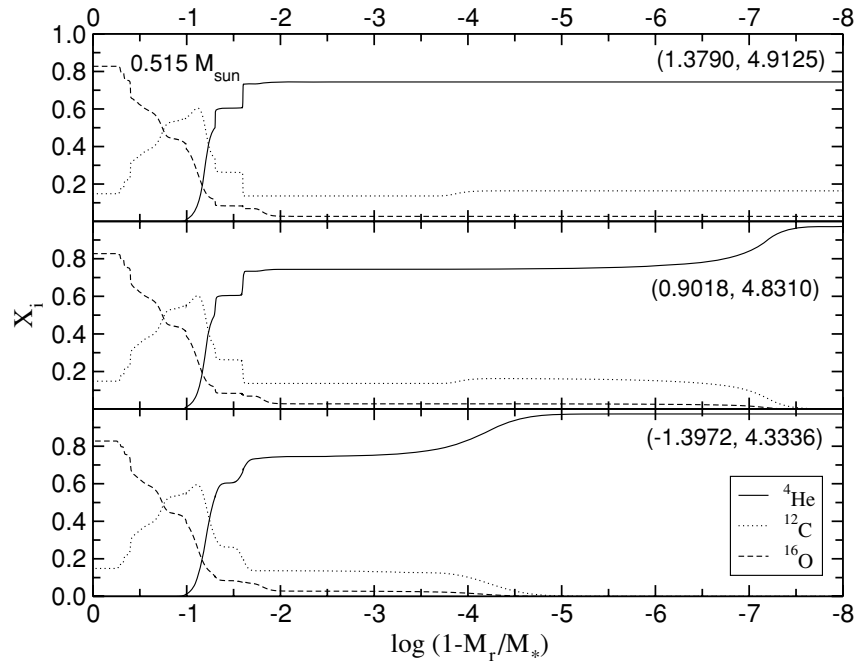

Figure 4. Same as Figure 3 but for the $0.515 M_{\odot}$ sequence.

proceeds, the He abundance in the outer layers increases, thus turning the PG 1159 star into a white dwarf of spectral type DO (Dehner \& Kawaler 1995; Gautschy \& Althaus 2002). At this point, we must mention that our treatment of diffusion cannot predict when a PG 1159 star is transformed into a DO white dwarf. To do this, a more elaborated treatment of diffusion in the surface layers than that we consider here-including mass loss through winds, and radiative levitation-should be used (Unglaub \& Bues 2000). Note the formation of a diffusively evolving double-layered chemical structure, that is, a pure He mantle and an intermediate remnant shell rich in He, carbon, and oxygen result of the last He thermal pulse. Because of the more intense gravitational field and the smaller He content in more massive remnants, the double-layered structure in our $0.87 M_{\odot}$ sequence almost disappears before the DBV instability strip is reached at $T_{\text {eff }} \approx 30,000 \mathrm{~K}$, see the bottom panel of Figure 3 . Indeed, the outer layer chemical profile of the massive remnant resembles that of a single-layered structure by the time cooling has proceeded to this stage. As shown by Fontaine \& Brassard (2002) and also by Althaus \& Córsico (2004), the presence of a double-layered structure bears consequences for the pulsational properties of variable DB white dwarfs. In particular, the double-layered structure causes the period-spacing diagrams to exhibit mode-trapping substructure (Althaus \& Córsico 2004). Thus, a complete assessment of the evolutionary history of $\mathrm{H}$-deficient white dwarf progenitors like that considered here constitutes a key aspect that has to be considered in asteroseismological studies of DBV stars. In this sense, we conclude that on the basis of a full account of progenitor evolution, a double-layered structure is not expected in $\mathrm{H}$-deficient white dwarfs with masses higher than about $0.87 M_{\odot}$ by the time evolution has proceeded to the domain of the DBV instability strip.

Our evolutionary sequences are appropriate for mass determinations of H-deficient white dwarfs, particularly at the early and hot stages when white dwarf evolution is still sensitive to the initial conditions. In Figure 5, we show the evolution of our sequences in the $\log g-\log T_{\text {eff }}$ diagram and the effective temperatures and surface gravities (in $\mathrm{cm} \mathrm{s}^{-2}$ ) derived by Hügelmeyer et al. (2006), Dreizler \& Werner (1996), and Dreizler et al. (1997) of the observed DO white dwarfs. They are listed in Table 3 together with the stellar masses determined by these authors using the evolutionary tracks of Wood

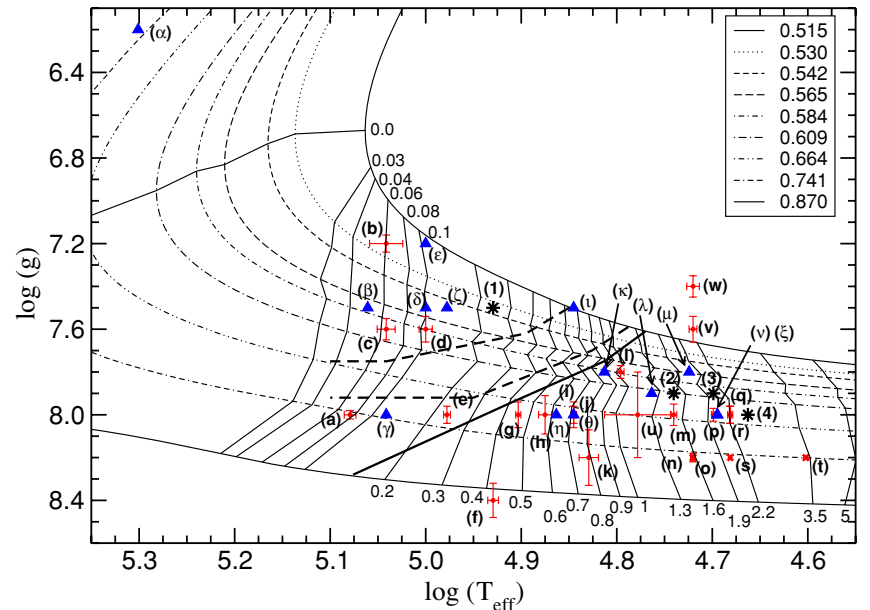

Figure 5. Surface gravity $g$ (in $\mathrm{cm} \mathrm{s}^{-2}$ ) as a function of $T_{\text {eff }}$ for our H-deficient sequences with thick He envelopes. From bottom to top, curves correspond to sequences with stellar masses from 0.870 to $0.515 M_{\odot}$. We also plot isochrones ranging from 0.03 to $5 \mathrm{Myr}$ measured from the highest effective temperature point. The observed DO white dwarfs analyzed by Hügelmeyer et al. (2006), Dreizler \& Werner (1996), and Dreizler et al. (1997), as listed in Table 3, are also shown (filled circles and triangles, and star symbols, respectively). The thick solid line corresponds to the wind limit for PG 1159 stars taken from Unglaub \& Bues (2000), and the two thick dashed lines to the wind limits for PG 1159 stars with $\mathrm{H} / \mathrm{He}=0.1$ and 0.01 (upper and lower line, respectively).

(A color version of this figure is available in the online journal.)

(1995) for carbon-core white dwarfs and an He layer mass of $10^{-4} M_{*}$ (Column 4). In passing, we note that the stellar mass of $\mathbf{J} 204158.98+000325.4$ listed in Hügelmeyer et al. (2006) is not correct, it should read 0.49 instead of $0.6 M_{\odot}$. We have corrected this in Table 3. From Figure 6, it is clear the importance of finite-temperature effects during the early evolutionary stages, particularly in the case of low-mass sequences. We expect then that the stellar mass for hot DOs with low gravities determined from cooling tracks that neglect the progenitor evolution will be different when our new evolutionary tracks are employed. Thus, we redetermined the stellar mass of the DO white dwarfs listed in Table 3 using our new sequences. The results are given in Column 5. For most DOs, mass determinations from both sets of tracks agree reasonably well, but in the case of the hot and lowest-gravity DOs, our evolutionary tracks lead to an increase in the derived stellar mass of up to $10 \%$ when compared with the determinations based on the cooling tracks of Wood (1995). We must caution, however, that some simplifications done in the calculations of Wood (1995), in particular regarding the chemical profile and He envelope mass of the models, prevent us from a consistent and meaningful comparison between both sets of tracks. For instance, the decrease in surface gravity resulting from the assumption of a pure carbon core in the models of Wood (1995) is in part compensated for by the lower He envelope mass that characterizes these models, as compared to our carbon-oxygen core white dwarf models that are characterized by more massive He envelopes. Finally, note that for the low-gravity DO, J075606.36+421613.0, we derive a stellar mass of $0.467 M_{\odot}$. Although this value results from extrapolation of our tracks, it is interesting to note that the resulting mass is substantially higher than the $0.42 M_{\odot}$ quoted by Hügelmeyer et al. (2006). The new stellar mass seems to suggest that the progenitor of this star could probably be an $\mathrm{He}-\mathrm{SdO}$ star formed in a delayed He-core flash (Miller Bertolami et al. 2008).

Our sequences provide also realistic evolutionary ages, particularly for the hot stages of white dwarf evolution, for which 
Table 3

Stellar Parameters of the DO White Dwarf Samples of Hügelmeyer et al. (2006), Dreizler \& Werner (1996), and Dreizler et al. (1997)

\begin{tabular}{|c|c|c|c|c|c|c|c|c|}
\hline DO Name & $\begin{array}{l}T_{\text {eff }} \\
(\mathrm{kK})\end{array}$ & $\begin{array}{c}\log g \\
\left(\mathrm{~cm} \mathrm{~s}^{-2}\right)\end{array}$ & $\begin{array}{c}M_{\mathrm{WD}} \\
\text { (Wood) }\end{array}$ & $\begin{array}{c}M_{\mathrm{WD}}\left(M_{\odot}\right) \\
\text { (Thick) }\end{array}$ & $\begin{array}{c}M_{\mathrm{WD}}\left(M_{\odot}\right) \\
(\text { Thin })\end{array}$ & $\begin{array}{c}\text { Age (Myr) } \\
\text { (Thick) }\end{array}$ & $\begin{array}{c}\text { Age (Myr) } \\
\text { (Thin) }\end{array}$ & $\overline{\operatorname{Ref}^{a}}$ \\
\hline J091433.61+581238.1 & $120.0 \pm 1.7$ & $8.00 \pm 0.02$ & 0.75 & $0.750 \pm 0.010$ & $0.740 \pm 0.010$ & $0.076 \pm 0.006$ & $0.074 \pm 0.006$ & $\overline{\text { (a) }}$ \\
\hline $\mathrm{J} 204158.98+000325.4$ & $110.0 \pm 4.5$ & $7.20 \pm 0.04$ & 0.49 & $0.525_{-0.002}^{+0.003}$ & $0.510_{-0.005}^{+0.007}$ & $0.060_{-0.010}^{+0.016}$ & $0.030_{-0.005}^{+0.007}$ & (b) \\
\hline $\mathrm{J} 214223.32+111740.4$ & $110.0 \pm 2.4$ & $7.60 \pm 0.05$ & 0.60 & $0.595_{-0.011}^{+0.012}$ & $0.580_{-0.013}^{+0.015}$ & $0.064_{-0.007}^{+0.008}$ & $0.054 \pm 0.006$ & (c) \\
\hline $\mathrm{J} 154752.33+423210.9$ & $100.0 \pm 1.6$ & $7.60 \pm 0.06$ & 0.59 & $0.584_{-0.019}^{+0.014}$ & $0.569_{-0.016}^{+0.017}$ & $0.107_{-0.009}^{+0.008}$ & $0.088 \pm 0.008$ & (d) \\
\hline $\mathrm{J} 151026.48+610656.9$ & $95.0 \pm 0.8$ & $8.00 \pm 0.04$ & 0.71 & $0.715 \pm 0.015$ & $0.708 \pm 0.016$ & $0.232 \pm 0.008$ & $0.233 \pm 0.008$ & (e) \\
\hline J084008.72+325114.6 & $85.0 \pm 1.1$ & $8.40 \pm 0.08$ & 0.90 & $0.900_{-0.045}^{+0.040}$ & $0.890 \pm 0.040$ & $0.420 \pm 0.015$ & $0.460 \pm 0.020$ & (f) \\
\hline J025403.75+005854.5 & $80.0 \pm 0.5$ & $8.00 \pm 0.06$ & 0.68 & $0.695 \pm 0.025$ & $0.688_{-0.021}^{+0.027}$ & $0.450 \pm 0.010$ & $0.455 \pm 0.010$ & (g) \\
\hline $\mathrm{J} 155356.81+483228.6$ & $75.0 \pm 1.2$ & $8.00 \pm 0.09$ & 0.68 & $0.687_{-0.037}^{+0.043}$ & $0.680_{-0.038}^{+0.043}$ & $0.565_{-0.030}^{+0.034}$ & $0.575 \pm 0.035$ & (h) \\
\hline J084223.14+375900.2 & $70.0 \pm 0.3$ & $8.00 \pm 0.06$ & 0.68 & $0.680_{-0.026}^{+0.027}$ & $0.673_{-0.026}^{+0.029}$ & $0.715 \pm 0.010$ & $0.725 \pm 0.010$ & (i) \\
\hline J140409.96+045739.9 & $70.0 \pm 0.5$ & $8.00 \pm 0.04$ & 0.68 & $0.680_{-0.018}^{+0.017}$ & $0.673_{-0.018}^{+0.019}$ & $0.715 \pm 0.015$ & $0.725 \pm 0.015$ & (j) \\
\hline $\mathrm{J} 113631.50+591229.8$ & $67.5 \pm 1.6$ & $8.20 \pm 0.13$ & 0.78 & $0.775_{-0.065}^{+0.070}$ & $0.770_{-0.057}^{+0.070}$ & $0.790 \pm 0.050$ & $0.820 \pm 0.050$ & (k) \\
\hline $\mathrm{J} 131724.75+000237.4$ & $62.5 \pm 0.5$ & $7.80 \pm 0.03$ & 0.58 & $0.582_{-0.012}^{+0.013}$ & $0.576_{-0.011}^{+0.012}$ & $1.040 \pm 0.020$ & $0.950 \pm 0.030$ & (1) \\
\hline J034101.39+005353.0 & $55.0 \pm 0.4$ & $8.00 \pm 0.05$ & 0.65 & $0.656 \pm 0.024$ & $0.650 \pm 0.025$ & $1.500 \pm 0.030$ & $1.500 \pm 0.030$ & $(\mathrm{~m})$ \\
\hline J003343.06+142251.5 & $52.5 \pm 0.2$ & $8.20 \pm 0.01$ & 0.76 & $0.755 \pm 0.005$ & $0.753 \pm 0.005$ & $1.570 \pm 0.010$ & $1.580 \pm 0.010$ & (n) \\
\hline J115218.69-024915.9 & $52.5 \pm 0.3$ & $8.20 \pm 0.02$ & 0.76 & $0.755 \pm 0.012$ & $0.753 \pm 0.011$ & $1.570 \pm 0.020$ & $1.580 \pm 0.020$ & (o) \\
\hline J034227.62-072213.2 & $50.0 \pm 0.2$ & $8.00 \pm 0.03$ & 0.65 & $0.647_{-0.014}^{+0.015}$ & $0.643 \pm 0.015$ & $1.970 \pm 0.020$ & $1.950 \pm 0.020$ & (p) \\
\hline J081115.09+270621.8 & $48.0 \pm 0.3$ & $8.00 \pm 0.04$ & 0.64 & $0.645 \pm 0.020$ & $0.640 \pm 0.020$ & $2.210 \pm 0.040$ & $2.190 \pm 0.040$ & (q) \\
\hline J113609.59+484318.9 & $48.0 \pm 0.1$ & $8.00 \pm 0.04$ & 0.64 & $0.645 \pm 0.020$ & $0.640 \pm 0.020$ & $2.210 \pm 0.020$ & $2.190 \pm 0.020$ & (r) \\
\hline $\mathrm{J} 081546.08+244603.3$ & $48.0 \pm 0.2$ & $8.20 \pm 0.01$ & 0.76 & $0.750 \pm 0.007$ & $0.747_{-0.005}^{+0.006}$ & $2.010 \pm 0.030$ & $2.000 \pm 0.020$ & (s) \\
\hline J015629.58+131744.7 & $40.0 \pm 0.2$ & $8.20 \pm 0.01$ & 0.75 & $0.741 \pm 0.007$ & $0.739_{-0.007}^{+0.006}$ & $3.400 \pm 0.050$ & $3.270 \pm 0.050$ & $(\mathrm{t})$ \\
\hline J091621.83+052119.2 & $60.0 \pm 5.0$ & $8.00 \pm 0.20$ & 0.66 & $0.664_{-0.087}^{+0.101}$ & $0.659_{-0.087}^{+0.103}$ & $1.170_{-0.260}^{+0.300}$ & $1.170_{-0.250}^{+0.300}$ & (u) \\
\hline $\mathrm{J} 133633.22-013116.5$ & $52.5 \pm 0.5$ & $7.60 \pm 0.06$ & 0.48 & $0.500 \pm 0.015$ & $0.485_{-0.015}^{+0.020}$ & $2.270 \pm 0.070$ & $2.400 \pm 0.100$ & (v) \\
\hline J075606.36+421613.0 & $52.5 \pm 0.8$ & $7.40 \pm 0.05$ & 0.42 & $0.467_{-0.003}^{+0.005}$ & $0.445 \pm 0.005$ & $2.900 \pm 0.150$ & $3.200 \pm 0.150$ & (w) \\
\hline KPD0005+5106 & 200 & 6.2 & $\cdots$ & 0.770 & 0.700 & -0.00153 & -0.00038 & $(\alpha)$ \\
\hline PG 0038+199 & 115 & 7.5 & 0.59 & 0.575 & 0.562 & 0.050 & 0.040 & $(\beta)$ \\
\hline PG 0109+111 & 110 & 8.0 & 0.74 & 0.735 & 0.730 & 0.120 & 0.120 & $(\gamma)$ \\
\hline PG 1034+001 & 100 & 7.5 & 0.56 & 0.554 & 0.545 & 0.100 & 0.080 & $(\delta)$ \\
\hline HS1830+7209 & 100 & 7.2 & 0.47 & 0.517 & 0.500 & 0.107 & 0.052 & $(\varepsilon)$ \\
\hline PG 0108+101 & 95 & 7.5 & 0.54 & 0.548 & 0.540 & 0.133 & 0.105 & $(\zeta)$ \\
\hline PG 0046+078 & 73 & 8.0 & 0.68 & 0.680 & 0.675 & 0.620 & 0.630 & $(\eta)$ \\
\hline PG $0237+116$ & 70 & 8.0 & 0.68 & 0.680 & 0.675 & 0.720 & 0.730 & $(\theta)$ \\
\hline RE0503-289 & 70 & 7.5 & 0.49 & 0.515 & 0.505 & 0.670 & 0.700 & ( \\
\hline HS0111+0012 & 65 & 7.8 & 0.58 & 0.587 & 0.580 & 0.880 & 0.820 & $(\kappa)$ \\
\hline Lanning 14 & 58 & 7.9 & 0.61 & 0.615 & 0.610 & 1.220 & 1.225 & $(\lambda)$ \\
\hline HZ21 & 53 & 7.8 & 0.56 & 0.562 & 0.555 & 1.750 & 1.750 & $(\mu)$ \\
\hline HS0103+2947 & 49.5 & 8.0 & 0.65 & 0.645 & 0.640 & 2.025 & 2.010 & (v) \\
\hline HD149499B & 49.5 & 8.0 & 0.65 & 0.645 & 0.640 & 2.025 & 2.010 & (v) \\
\hline MCT 2148-294 & 85 & 7.5 & 0.53 & 0.536 & 0.529 & 0.240 & 0.210 & (1) \\
\hline PG 0929+270 & 55 & 7.9 & 0.61 & 0.608 & 0.603 & 1.450 & 1.460 & (2) \\
\hline PG 1057-059 & 50 & 7.9 & 0.60 & 0.601 & 0.595 & 2.025 & 1.980 & (3) \\
\hline PG $1133+489$ & 46 & 8.0 & 0.64 & 0.640 & 0.635 & 2.500 & 2.470 & (4) \\
\hline
\end{tabular}

Notes. Column 4 gives the stellar mass in solar units as derived by these authors using the cooling tracks of Wood (1995). Columns 5 and 6 list, respectively, the stellar mass in solar units derived from our evolutionary tracks with thick and thin He envelopes. The next two columns give the white dwarf ages. The effective temperature and gravity of KPD0005+5106 have been taken from Werner et al. (2008b). For this star, the lower limit to log $g$ has been adopted. The zero point for the ages is at the highest effective temperature.

${ }^{a}$ Roman letters: Hügelmeyer et al. (2006); Greek letters: Dreizler \& Werner (1996); Numbers: Dreizler et al. (1997).

employing artificial procedures to generate starting white dwarf configurations may lead to evolutionary sequences with erroneous thermomechanical structures and, thus, to wrong ages. We employed our tracks to derive evolutionary ages for the white dwarfs plotted in Figure 5. They are listed in Column 7 of Table 3. The zero-point age corresponds to the highest effective temperature for each track, see Figure 2. For the stellar masses not covered by our tracks, ages were obtained from extrapolation, so they should be taken with some caution. We also plot isochrones in Figure 5 for better visualization. Note that the bulk of observed DO white dwarfs have ages between 0.6 and 2.5 Myr, though the hottest members of the sample are indeed very young, with ages less than $0.10 \mathrm{Myr}$. Note also that the mass distribution of young DO white dwarfs differs considerably from that of the older ones. In fact, for ages less than about $\sim 0.4 \mathrm{Myr}, \mathrm{DO}$ white dwarfs less massive than $\approx 0.6 M_{\odot}$ are $75 \%$ of the total number of DOs at those ages, whilst for longer ages, this contribution declines to $40 \%$. Finally, the population of high-gravity DOs increases considerably for ages greater than $0.4 \mathrm{Myr}$, below $T_{\text {eff }} \approx 85,000 \mathrm{~K}$. These features 


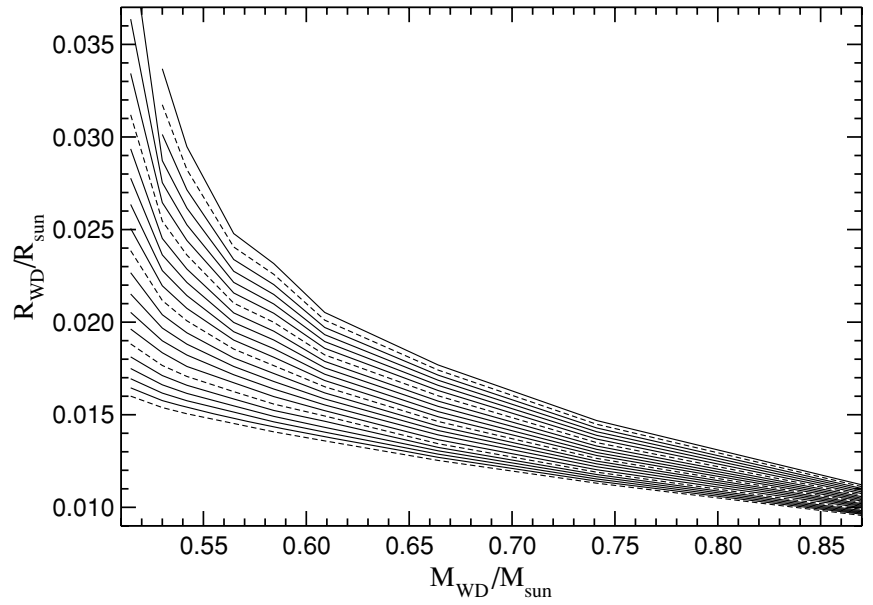

Figure 6. Mass-radius relations for our H-deficient sequences with thick $\mathrm{He}$ envelopes, corresponding to several effective temperatures. From bottom to top, we show relations for 40,000-124,000 K in temperature steps of $4000 \mathrm{~K}$. In the interest of clarity, the mass-radius relations for effective temperatures $40,000 \mathrm{~K}$, $60,000 \mathrm{~K}, 80,000 \mathrm{~K}, 100,000 \mathrm{~K}$, and $120,000 \mathrm{~K}$ are displayed with dashed lines.

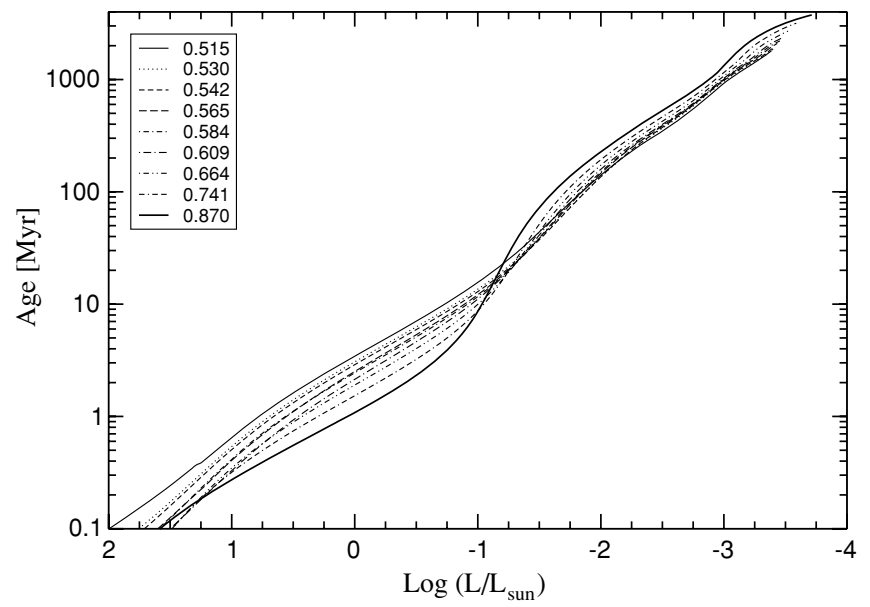

Figure 7. Age (in Myr) vs. luminosity (in solar units) for our H-deficient white dwarf sequences.

may help to shed light on the formation and evolutionary status of DOs.

In Figure 7, we show the age (Myr) as a function of the luminosity (in solar units) for all the H-deficient sequences. At very high luminosity stages, residual He shell burning constitutes an appreciable fraction of the surface luminosity. For instance, at $\log T_{\text {eff }}=5$, the He shell burning luminosity represents $25 \%$ of the surface luminosity in our lowest-mass sequence. This contribution declines steeply to $10 \%$ at $\log T_{\text {eff }}=$ 4.95. Except for these very high luminosity stages, evolution during the early stages is driven by neutrino emission and the release of gravothermal energy. In particular, neutrino losses exceed photon luminosity during most of the hot white dwarf stages. Indeed, except for the hot and low-gravity DO sample shown in Figure 5, it is during this "neutrino epoch" that most DO white dwarfs are observed. Note from Figure 7 that the imprints of neutrino emission on the cooling curve are more noticeable as the mass increases. Less massive sequences are older than more massive sequences for $\log \left(L / L_{\odot}\right) \gtrsim-1$. At this stage, neutrino emission arrives at its end, thus causing a change in the slope in the cooling curve around $\log \left(L / L_{\odot}\right) \sim-1$, and later for the less massive sequences. At lower luminosities,

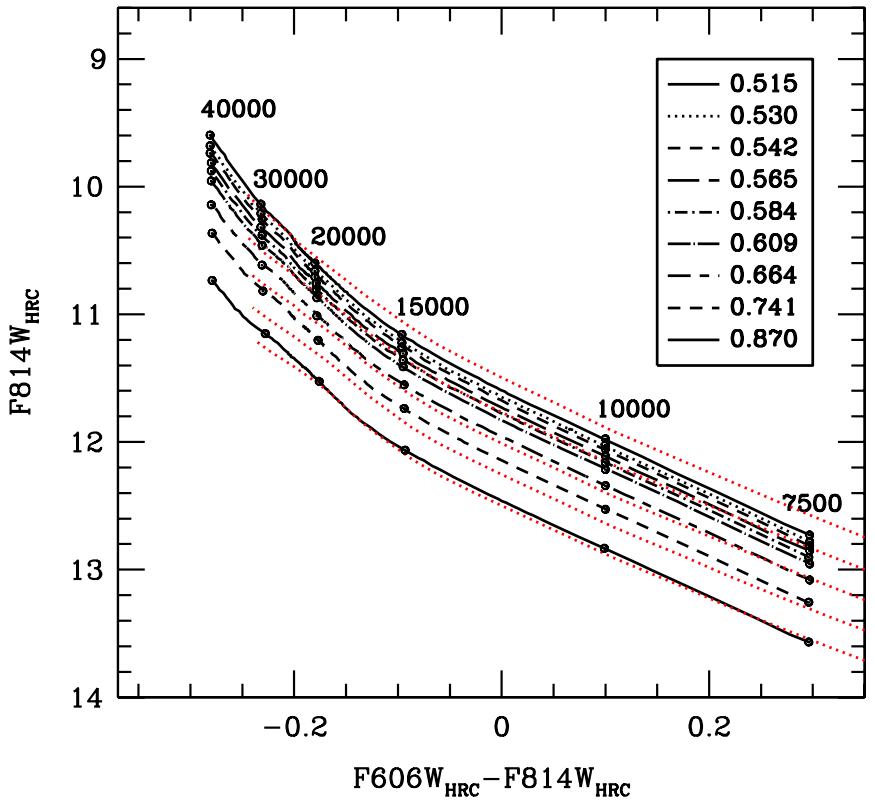

Figure 8. F814W vs. F606W-F814W color-magnitude diagram for our DB white dwarf sequences with pure He envelopes. Dotted lines correspond to Bergeron et al. (1995) sequences for He-atmospheres white dwarfs and stellar masses of, from top to bottom, 0.5, 0.6, 0.7, 0.8, and $0.9 M_{\odot}$.

(A color version of this figure is available in the online journal.)

Coulomb interactions become more important, increasing the specific heat, until crystallization and the associated release of latent heat start at the center (Van Horn 1968; Winget et al. 2009). At this point, cooling can be well understood on the basis of the simple cooling theory of Mestel (1952), which predicts less massive white dwarfs to cool faster.

For all our cooling sequences, we computed accurate colors and magnitudes based on non-gray local thermodynamic equilibrium (LTE) model atmospheres (Rohrmann et al. 2002). The calculations were done for a pure helium composition and for the HST ACS filters (Vega-mag system) and UBVRI photometry. Briefly, each model atmosphere is approximated, locally, by plane-parallel layers in hydrostatic equilibrium and LTE. Convective energy transport in cooler atmospheres has been calculated with the so-called ML2 parameterization of the mixing-length theory. The correct total energy flux transported by radiation and convection is obtained from a Rybicki scheme, see details in Rohrmann et al. (2002). Chemical populations are computed within the occupation probability formalism of Hummer \& Mihalas (1988). For helium pure models, the opacity sources include bound-free $\left(\mathrm{He}, \mathrm{He}^{+}, \mathrm{He}^{2+}\right)$ and free-free $\left(\mathrm{He}, \mathrm{He}^{+}, \mathrm{He}^{2+}, \mathrm{He}^{-}\right)$transitions, electronic and Rayleigh $(\mathrm{He})$ scattering, and the most significant line series of $\mathrm{He}$ and $\mathrm{He}^{+}$. The level occupation probabilities are explicitly included in the evaluation of the line and continuum opacities. As an example of the present atmosphere calculations, we show in Figure 8 the F814W, F606W-F814W color-magnitude diagram for all our sequences. Colors have been computed for effective temperatures lower than 40,000 K, because NLTE effects become important above this temperature. For higher effective temperatures, most of radiation flux emerges at shorter wavelengths. We then expect colors to present a markedly less dependence with temperature. Comparison between our color sequences and those due to Bergeron (see Figure 8) reveals a rather good agreement, with some small discrepancies presumably due to differences of the constitutive physics used in the atmosphere codes. 


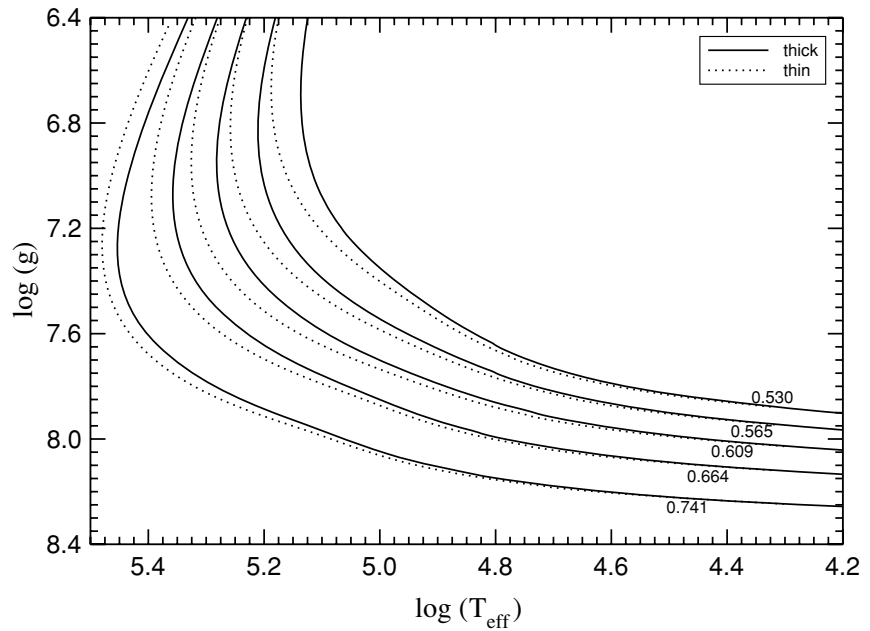

Figure 9. Surface gravity as a function of $T_{\mathrm{eff}}$ for the sequences with thin and thick envelopes and stellar masses of $0.530,0.565,0.609,0.664$, and $0.741 M_{\odot}$

\subsection{Thin Envelope Sequences}

In Section 3.1, we studied sequences characterized by the thickest He-rich envelope that can be left after a born-again episode. However, there is theoretical and observational evidence that He-rich envelopes could be substantially thinner. For instance, using asteroseismology, Kawaler \& Bradley (1994) derived an He-rich envelope of $\sim 10^{-3} M_{\odot}$ in at least one of the PG 1159 stars with masses $0.6 M_{\odot}$. More recently, Althaus et al. (2008) showed that PG 1159 star models with thin He-rich envelopes appear to be needed to solve the discrepancy between the observed (Costa \& Kepler 2008) and the theoretical rates of period change in the pulsating star PG 1159-035. A more direct evidence for the existence of PG 1159 stars with thin $\mathrm{He}$ envelopes is provided by the strong mass-loss events reported in the post-born-again star V4334 Sgr, also known as Sakurai's object (van Hoof et al. 2007). All these facts point at the possibility that some PG 1159 stars could be characterized by He-rich envelopes thinner than expected from the standard theory of stellar evolution (Althaus et al. 2009a). Last but not least, the mere existence of the hot DQ class of white dwarfs (Dufour et al. 2007, 2008) could be reflecting the existence of He-rich white dwarfs with extremely thin He envelopes (Dufour et al. 2008; Althaus et al. 2009b). In view of these considerations, we examined the evolution of $\mathrm{H}$-deficient white dwarfs for the extreme situation in which a very thin He envelope could result from prior evolution. To this end, we computed five sequences with stellar masses of $0.53,0.565,0.609,0.664$, and $0.741 M_{\odot}$ characterized by He-rich envelopes with masses $\sim 10^{-8} M_{*}$, a value which is of the order required by the diffusive/convective mixing scenario to explain the origin of hot DQ white dwarfs (Althaus et al. 2009b). To construct initial models with such thin He-rich envelopes, we artificially reduced the He content of our models to $\sim 10^{-8} M_{*}$ well before they reach the PG 1159 domain. These sequences were evolved down to $T_{\text {eff }} \approx 20,000 \mathrm{~K}$, where the He-rich envelope is diluted by the outer convection zone.

In Figure 9, the resulting tracks in the $\log g-\log T_{\text {eff }}$ plane are compared with the sequences corresponding to thick $\mathrm{He}$ envelope models analyzed in Section 3.1. When thin He-rich envelopes are considered, the evolutionary tracks in this plane are strongly modified for the PG 1159 regime, with important consequences for PG 1159 spectroscopic mass determinations

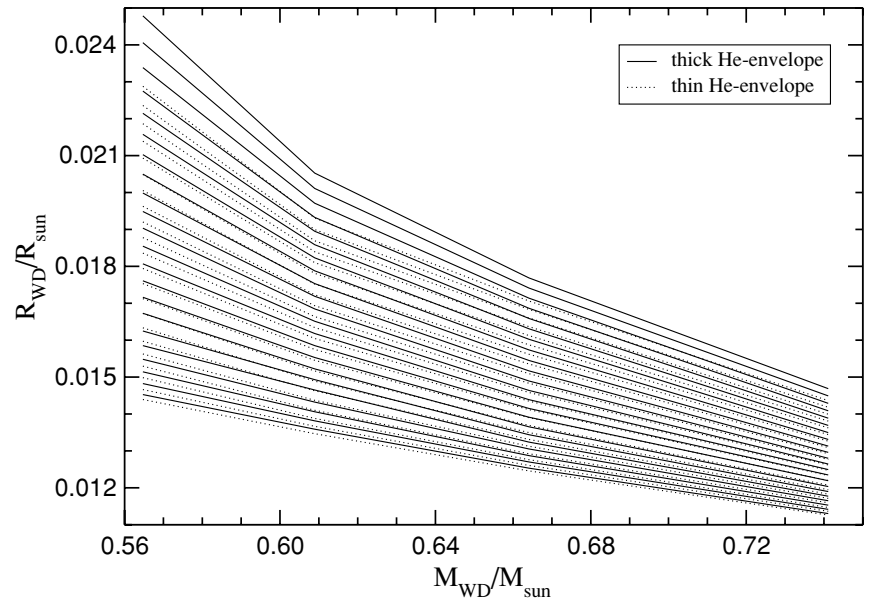

Figure 10. Mass-radius relations for $\mathrm{H}$-deficient sequences with thick (solid lines) and thin (dotted line) He envelopes. Curves correspond to effective temperatures of, from bottom to top, 40,000-124,000 K in temperature steps of $4000 \mathrm{~K}$

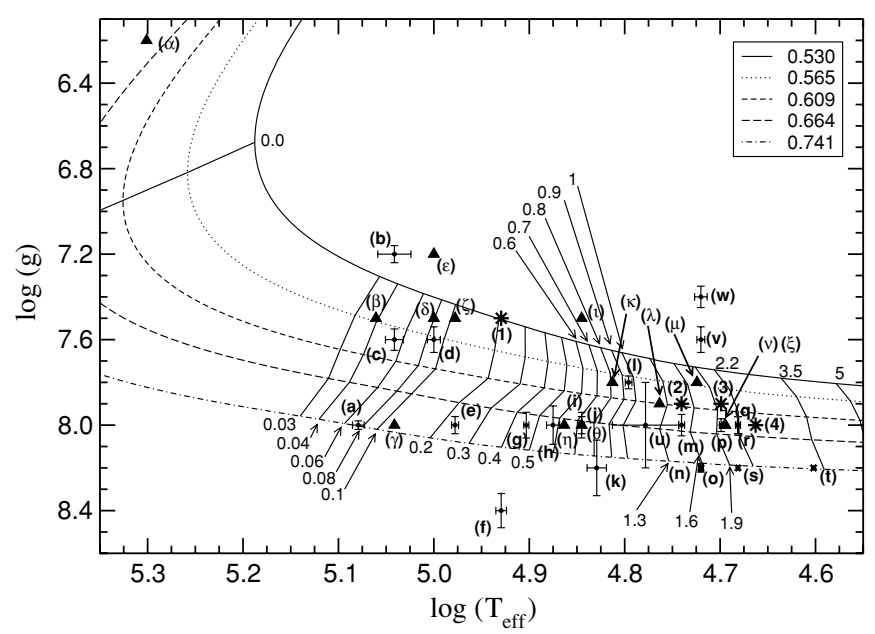

Figure 11. Same as Figure 5 for our H-deficient sequences with thin $\mathrm{He}$ envelopes. We also plot isochrones (Myr) ranging from 0.03 to $5 \mathrm{Myr}$. The location of the observed DO white dwarfs listed in Table 3 is also shown.

(Althaus et al 2008). For the white dwarf regime, models with thin He envelopes are more compact—see Figure 10—and, thus, are characterized by higher gravities. As a result, lower white dwarf masses would be inferred when the sequences with thin He envelopes are used, as it is clear by examining Column 6 in Table 3. From Figure 9, we expect this to be relevant only for the hot white dwarf stages. Indeed, note from Table 3 that only for hot DO white dwarfs we find a noticeable, albeit small, change in the stellar mass when the models with thin He-rich envelopes are used. In Figure 11, we show the white dwarf tracks for thin He-rich envelopes together with the observed DO white dwarfs as listed in Table 3. Note that the low-mass sequences with thin $\mathrm{He}$ envelopes evolve faster during the hot stages of evolution. In fact, for these DO white dwarfs significantly smaller ages are inferred when the tracks with thin He envelopes are used, see Column 8 in Table 3. This is because the He shell burning does not represent an energy source in the sequences with thin $\mathrm{He}$ envelopes. Finally, the formation of a double-layered structure in pulsating DB stars is not expected if $\mathrm{H}$-deficient white dwarfs are formed with sufficiently thin He-rich envelopes. 


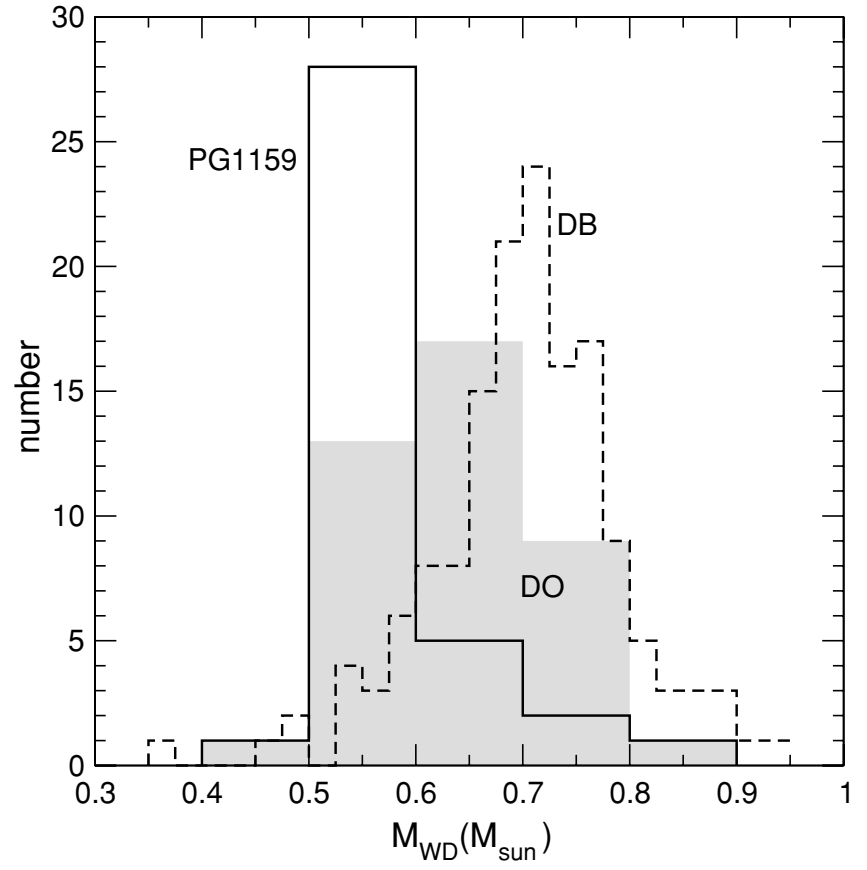

Figure 12. Shaded histogram shows the mass distribution for the DO white dwarfs listed in Table 3. The mass determinations have been done using the evolutionary tracks described in this work. The solid line corresponds to the PG 1159 mass distribution from Miller Bertolami \& Althaus (2006), and the dashed line to that of DB white dwarfs taken from Kepler et al. (2007).

\section{DISCUSSION AND CONCLUSIONS}

Motivated by the increasing number of detected hot $\mathrm{H}$ deficient white dwarfs (of the DO type) as well as by the lack of realistic sets of evolutionary tracks appropriate for their interpretation, we computed new evolutionary sequences and colors for non-DA white dwarfs with stellar masses of $0.515,0.530,0.542,0.565,0.584,0.609,0.664,0.741$, and $0.870 M_{\odot}$. In contrast to previous studies, these sequences were obtained considering the full evolution of the progenitor stars, from the ZAMS through the thermally pulsing AGB phase and born-again episode, to the domain of the PG 1159 stars-see Miller Bertolami \& Althaus (2006) for details. Our H-deficient white dwarf evolutionary tracks are thus not affected by inconsistencies arising from artificial procedures to generate the starting white dwarf configurations, and so, they are appropriate for realistic mass and age determinations of $\mathrm{H}$-deficient white dwarfs, particularly for objects at the early and hot stages. Calculations were followed to advanced phases of evolution down to an effective temperature of $7000 \mathrm{~K}$.

We applied this new set of tracks to redetermine the stellar masses of all known DO white dwarfs with spectroscopically determined effective temperatures and gravities. The results are listed in Table 3. The stellar masses for hot DOs with the lowest gravities inferred from our tracks differ appreciably from the determinations based on the evolutionary tracks of Wood (1995). This is because these tracks do not consider the full evolution of progenitor stars. This is also true for the age determinations of these stars. In particular, our evolutionary sequences provide realistic ages at very early stages of evolution. For thick He layers, we find that He shell burning supplies an appreciable fraction of the surface luminosity, thus changing appreciably the evolutionary timescales, particularly of the hottest DO white dwarfs with low gravities. The amount of He left in the remnant white dwarf affects both the mass and the age determinations of hot DOs. Models with thin He-rich envelopes are characterized by higher gravities than models with thick He-rich envelopes, thus predicting lower white dwarf stellar masses for the observed DOs.

The evolutionary tracks presented here cover all the stages from the domain of luminous PG 1159 star region to the hot white dwarf domain. Thus, they constitute a homogeneous set of evolutionary tracks to study both type of stars. This is of major interest because we can make consistent mass determinations for both the DOs and PG 1159 stars for the first time. In Figure 12, we compare the mass distribution of the DO white dwarfs listed in Table 3 with the mass distribution of PG 1159 stars constructed on the basis of mass determinations made by Miller Bertolami \& Althaus (2006), shaded region and solid line, respectively. The size of the bins in both distributions is $0.1 M_{\odot}$. Note that massive DOs outnumber massive PG 1159 stars. We obtain a mean DO mass of $0.644 M_{\odot}$, considerably higher (by $0.071 M_{\odot}$ ) than the mean mass of PG $1159 \mathrm{stars}, 0.573 M_{\odot}$. One may wonder if the reason for this difference is simply that evolution through the PG 1159 stage proceeds considerably faster for massive stars (Miller Bertolami \& Althaus 2006), thus decreasing the possibility of detection. To elaborate on this point, we have proceeded as follows. In order to avoid the possibility that the massive PG 1159 stars are underrepresented because they do not spend long times in the PG 1159 stage, we have tested the hypothesis that PG 1159 stars and DO white dwarfs form an isolated evolutionary sequence by analyzing the PG $1159+$ DO group as a whole. Taking advantage of the consistent set of tracks for both groups, we have divided the sample (78 stars) into two groups based on their age. The young group, which consists of all stars with ages below $0.3 \mathrm{Myr}$ (this includes all 37 PG 1159 stars plus 12 young DO white dwarfs) and the old group consisting of all DO white dwarfs with ages greater than 0.4 Myr (29 stars). It must be noted that there are no PG 1159 stars in the old group.

The choice of the 0.3-0.4 Myr isochrone to divide both groups is admittedly arbitrary but suggested by the location of the different wind limits studied by Unglaub \& Bues (2000). Dividing the whole PG $1159+$ DO group by an isochrone allows to test the hypothesis that PG 1159 and DO stars form an isolated evolutionary sequence. This can be done comparing their mass distributions (as relative numbers within a given group will not be affected by differences in evolutionary speeds). The two groups show significant differences in their mass distributions. While the young group has a mean mass of $M_{\mathrm{Y}}=0.584 M_{\odot}$ (with a variance of $\sigma_{\mathrm{Y}}=0.080 M_{\odot}$ ) the old group has a mean mass of $M_{\mathrm{O}}=0.655 M_{\odot}$ (with a variance of $\sigma_{\mathrm{O}}=0.087 M_{\odot}$ ). Thus, the difference in the mean masses is comparable with the variance within each sample, suggesting that both mass distributions are significantly different. This is confirmed by a Kolmogorov-Smirnov test. The result of this test is that the probability that both samples are taken from the same mass distribution is rather small $\left(\sim 10^{-5}\right)$.

These results clearly suggest that there may be some other important evolutionary channels operating within PG 1159 and DO stars. For instance, it is possible that not all PG 1159 stars evolve to DOs. In particular, those PG 1159 stars resulting from a LTP_low-mass remnants are more prone to experience a LTP episode-are expected to evolve into DA white dwarfs (near the winds limits shown in Figure 5 with solid dashed lines), thus avoiding the DO stage, as shown by Unglaub \& Bues (2000). This is so because, in contrast to a VLTP event, in a LTP, H 
is not burned, but instead diluted to low surface abundances (Miller Bertolami \& Althaus 2006). Alternatively, some DO white dwarfs could result from evolutionary channels that not involve only the PG 1159 stars. For instance, they could be the result of post-merger evolution involving the giant, H-deficient $\mathrm{RCrB}$ stars, via the evolutionary link $\mathrm{RCrB} \rightarrow \mathrm{EHe}$ (extreme $\mathrm{He}$ stars $) \rightarrow \mathrm{He}_{-} \mathrm{SdO}^{+} \rightarrow \mathrm{O}(\mathrm{He}) \rightarrow$ DO-see Rauch et al. (2006) for a connection between $\mathrm{O}(\mathrm{He})$ stars and $\mathrm{RCrB}$ stars. This is reinforced by the recent study by Werner et al. (2008a, 2008b) of the star KPD0005+5106, the hottest known DO white dwarf. These authors present evidence that KPD0005+5106 is not a descendant of PG 1159 stars, but more probably related to the $\mathrm{O}(\mathrm{He})$ stars and $\mathrm{RCrB}$ stars.

The group of young and hot DO white dwarfs is particularly relevant. Note that they are located considerably above the wind limit for PG 1159 stars (see Figure 5). In fact, above this line, mass loss is large enough to prevent gravitational settling. Hence, the transformation of a PG 1159 star into a DO white dwarf should be expected approximately below this line. Although the estimated mass-loss rates could be overestimated by more than a factor of 10 - in which case the transition from PG 1159 to DO would be near a line with $\log g \approx 7.5$ (Unglaub $\&$ Bues 2000) - it could be possible that the less massive and young DOs are not the descendants of PG 1159 stars. This is also suggested by the fact that for ages less than about 0.3-0.4 Myr, the DO white dwarf population is markedly less massive than at greater ages. Indeed, the number of detected old, less massive DOs is smaller than the number of young, less massive DOs, despite the evolutionary timescales being a factor about 5 for the former. This could be indicative that some of the less massive DOs experience a transformation in their spectral type after $\approx 0.3-0.4 \mathrm{Myr}$ of evolution. In particular, traces of $\mathrm{H}$ could turn the white dwarf spectral type from DO to DA as a result of gravitational settling. An approximate location where this transformation should occur is provided by the thick dashed lines shown in Figure 5, which provides the wind limits for PG 1159 stars with different initial H abundances (Unglaub \& Bues 2000). It is worth mentioning that because of the much smaller carbon content and correspondingly less intense winds, such limits would be expected at lower gravities in a DO white dwarf with similar $\mathrm{H}$ content. According to Unglaub \& Bues (1998), for a DO white dwarf with $\log g=7$ and $\mathrm{H}$ number ratio of 0.01 , the transformation into a DAO in presence of a moderate mass loss would take about $0.25 \mathrm{Myr}$, not very different from the age range where the DO mass distribution seems to change. At this point, it is worthwhile to comment on the possibility that the origin of low-mass, hot DOs can be connected with the merger of two low-mass, He-core white dwarfs (Guerrero et al. 2004; Lorén-Aguilar et al. 2005), which will evolve to become a low-gravity extreme He star and then a hot subdwarf (Saio \& Jeffery 2000). The presence of $\mathrm{H}$ expected in this case could lead to a transformation of the DO white dwarfs into DA ones.

In Figure 12, we have also included with dashed line the mass distribution of the DB white dwarfs in SDSS hotter than $T_{\text {eff }}=16,000 \mathrm{~K}$ taken from Kepler et al. (2007). The $\mathrm{DB}$ distribution is more weighted to massive stars. In fact, the DB mean mass is of $0.711 M_{\odot}$, considerably higher than the mean DO mass of $0.644 M_{\odot}$. This marked difference in the mass distribution between both populations is certainly quite unexpected in view of our understanding that DO stars must evolve into DB stars. However, it is possible that the DB stellar masses considered in Figure 12 could be overestimated. This hypothesis was raised by Kepler et al. (2007), who discuss that an increase in the measured surface gravity of DBs below $T_{\text {eff }} \approx 18,000 \mathrm{~K}$ could result from missing or incorrect physics in model atmospheres at such temperatures. It could be also possible that the increase in the gravity of the DBs by $T_{\text {eff }} \approx 18,000 \mathrm{~K}$ could be reflecting the existence of DBs with traces of carbon in their atmospheres. As shown by Dufour et al. (2005) in the context of cool DQs, the surface gravities derived from model atmospheres including carbon are significantly lower than the gravities obtained from pure He models. The idea that some carbon could be present in the atmosphere of some hot DBs is particularly attractive if a large fraction of DB white dwarfs evolve through the hot DQ stage. Indeed, as discussed in Althaus et al. (2009b) in the diffusive/convective mixing picture for the hot DQ formation, shortly after the formation of the carbon-rich convective envelope, by $T_{\text {eff }} \approx 20,000 \mathrm{~K}$, a substantial depletion of carbon is expected in the entire convective envelope, because of the very short diffusion timescale at the base of the convection zone. Hence, it is expected that the hot DQ stage, during which the white dwarf is characterized by high amounts of carbon in its surface, is indeed a short-lived phase. However, the change in composition in the convective envelope will lead to changes in the depth of the convection zone, which will affect, in turn, the timescales of diffusion. The lack of detailed computations of this feedback between convection and diffusion shortly after the formation of a DQ does not allow to make reasonable predictions, but it is not discarded that the star experiences additional mixing episodes, eventually reaching some stationary situation in which the star resembles an He-rich DB white dwarf with some carbon in its atmosphere. This being the true course of events, it would be worthwhile to redetermine the mass distribution of DBs in the frame of model atmospheres that incorporate the effects of the presence of carbon. Finally, in view of the material discussed in this section, it is feasible that a fraction of low-mass DOs experiences a change in their spectral type at young ages.

In closing, because our DO tracks consider the evolutionary history of progenitor, we can make sound predictions about the chemical profile expected in the envelope of $\mathrm{H}$-deficient white dwarfs. This is of relevance for asteroseismological inferences about pulsating DB white dwarfs. In particular, we find that depending on the stellar mass, diffusion processes lead to a variety of envelope chemical profiles, from singlelayered to double-layered structures, by the time evolution has proceeded to the domain of the variable DBs. Detailed tabulations of our evolutionary sequences are available at our Web site http://www.fcaglp.unlp.edu.ar/evolgroup.

This research was supported by AGENCIA: Programa de Modernización Tecnológica BID 1728 OC-AR, by the AGAUR, by MCINN grant AYA2008-04211-C02-01, by the European Union FEDER funds, and by PIP 6521 grant from CONICET. L.G.A. also acknowledges a PIV grant of the AGAUR of the Generalitat de Catalunya. We also acknowledge the comments and suggestions of our referee, K. Werner, which strongly improved the original version of this work. This research has made use of NASA's Astrophysics Data System. Finally, we thank H. Viturro and R. Martínez for technical support

\section{REFERENCES}

Abazajian, K., et al. 2003, AJ, 126, 2081

Adelman-McCarthy, J. K., et al. 2006, ApJS, 162, 38

Althaus, L. G., \& Córsico, A. H. 2004, A\&A, 417, 1115

Althaus, L. G., Córsico, A. H., \& Miller Bertolami, M. M. 2007a, A\&A, 467, 1175 
Althaus, L. G., Córsico, A. H., Miller Bertolami, M. M., García-Berro, E., \& Kepler, S. O. 2008, ApJ, 677, L35

Althaus, L. G., Córsico, A. H., Torres, S., \& García-Berro, E. 2009a, A\&A, 494 1021

Althaus, L. G., García-Berro, E., Córsico, A. H., Miller Bertolami, M. M., \& Romero, A. D. 2009b, ApJ, 693, L23

Althaus, L. G., García-Berro, E., Isern, J., Córsico, A. H., \& Rohrmann, R. D. 2007b, A\&A, 465, 249

Althaus, L. G., Panei, J. A., Romero, A. D., Rohrmann, R. D., Córsico, A. H., García-Berro, E., \& Miller Bertolami, M. M. 2009c, A\&A, 502, 207

Althaus, L. G., Serenelli, A. M., Córsico, A. H., \& Montgomery, M. H. 2003, A\&A, 404, 593

Althaus, L. G., Serenelli, A. M., Panei, J. A., Córsico, A. H., García-Berro, E., \& Scóccola, C. G. 2005, A\&A, 435, 631

Angulo, C., et al. 1999, Nucl. Phys. A, 656, 3

Benvenuto, O. G., \& Althaus, L. G. 1999, MNRAS, 303, 30

Bergeron, P., Wesemael, F., \& Beauchamp, A. 1995, PASP, 107, 1047

Blöcker, T. 1995, A\&A, 297, 727

Burgers, J. M. 1969, Flow Equations for Composite Gases (New York: Academic)

Cassisi, S., Potekhin, A. Y., Pietrinferni, A., Catelan, M., \& Salaris, M. 2007, ApJ, 661, 1094

Caughlan, G. R., \& Fowler, W. A. 1988, At. Data Nucl. Data Tables, 40, 283

Córsico, A. H., Althaus, L. G., \& Miller Bertolami, M. M. 2006, A\&A, 458, 259

Córsico, A. H., Benvenuto, O. G., Althaus, L. G., Isern, J., \& García-Berro, E. 2001, New Astron., 6, 197

Costa, J. E. S., \& Kepler, S. O. 2008, A\&A, 489, 1225

Dehner, B. T., \& Kawaler, S. D. 1995, ApJ, 445, L141

Díaz-Pinto, A., García-Berro, E., Hernanz, M., Isern, J., \& Mochkovitch, R. 1994, A\&A, 282, 86

Dreizler, S., \& Heber, U. 1998, A\&A, 334, 618

Dreizler, S., \& Werner, K. 1996, A\&A, 314, 217

Dreizler, S., Werner, K., Heber, U., Reid, N., \& Hagen, H. 1997, in The Third Conference on Faint Blue Stars, ed. A. G. D. Philip et al. (New York: L. Davis Press), 303

Dufour, P., Bergeron, P., \& Fontaine, G. 2005, ApJ, 627, 404

Dufour, P., Fontaine, G., Liebert, J., Schmidt, G. D., \& Behara, N. 2008, ApJ, 683, 978

Dufour, P., Liebert, J., Fontaine, G., \& Behara, N. 2007, Nature, 450, 522

Eisenstein, D. J., et al. 2006, ApJS, 167, 40

Fontaine, G., \& Brassard, P. 2002, ApJ, 581, L33

Fontaine, G., \& Brassard, P. 2008, PASP, 120, 1043

Fujimoto, M. Y. 1977, PASJ, 29, 331

García-Berro, E., Hernanz, M., Isern, J., \& Mochkovitch, R. 1988, Nature, 333, 642

Gautschy, A., \& Althaus, L. G. 2002, A\&A, 382, 141

Guerrero, J., García-Berro, E., \& Isern, J. 2004, A\&A, 413, 257

Haft, M., Raffelt, G., \& Weiss, A. 1994, ApJ, 425, 222

Hansen, B. M. S., et al. 2007, ApJ, 671, 380

Herwig, F., Blöcker, T., Langer, N., \& Driebe, T. 1999, A\&A, 349, L5

Hügelmeyer, S. D., Dreizler, S., Homeier, D., Krzesiński, J., Werner, K., Nitta, A., \& Kleinman, S. J. 2006, A\&A, 454, 617

Hügelmeyer, S. D., Dreizler, S., Werner, K., Krzesiński, J., Nitta, A., \& Kleinman, S. J. 2005, A\&A, 442, 309

Hummer, D. G. 1988, ApJ, 331, 794
Iben, I., Jr., Kaler, J. B., Truran, J. W., \& Renzini, A. 1983, ApJ, 264, 605 Iglesias, C. A., \& Rogers, F. J. 1996, ApJ, 464, 943

Isern, J., García-Berro, E., Hernanz, M., Mochkovitch, R., \& Torres, S. 1998, ApJ, 503, 239

Isern, J., García-Berro, E., Torres, S., \& Catalán, S. 2008, ApJ, 682, L109

Itoh, N., Hayashi, H., Nishikawa, A., \& Kohyama, Y. 1996, ApJS, 102, 411

Kawaler, S. D., \& Bradley, P. A. 1994, ApJ, 427, 415

Kepler, S. O., Kleinman, S. J., Nitta, A., Koester, D., Castanheira, B. G., Giovannini, O., Costa, A. F. M., \& Althaus, L. 2007, MNRAS, 375, 1315

Kleinman, S. J., et al. 2004, ApJ, 607, 426

Krzesiński, J., Nitta, A., Kleinman, S. J., Harris, H. C., Liebert, J., Schmidt, G., Lamb, D. Q., \& Brinkmann, J. 2004, A\&A, 417, 1093

Lorén-Aguilar, P., Guerrero, J., Isern, J., Lobo, J. A., \& García-Berro, E. 2005, MNRAS, 356, 627

Magni, G., \& Mazzitelli, I. 1979, A\&A, 72, 134

Mestel, L. 1952, MNRAS, 112, 583

Miller Bertolami, M. M., \& Althaus, L. G. 2006, A\&A, 454, 845

Miller Bertolami, M. M., Althaus, L. G., Serenelli, A. M., \& Panei, J. A. 2006, A\&A, 449, 313

Miller Bertolami, M. M., Althaus, L. G., Unglaub, K., \& Weiss, A. 2008, A\&A, 491,253

O'Brien, M. S., \& Kawaler, S. D. 2000, ApJ, 539, 372

Prada Moroni, P. G., \& Straniero, O. 2007, A\&A, 466, 1043

Rauch, T., Reiff, E., Werner, K., Herwig, F., Koesterke, L., \& Kruk, J. W. 2006, in ASP Conf. Ser. 348, Astrophysics in the Far Ultraviolet: Five Years of Discovery with FUSE, ed. G. Sonneborn, H. Moos, \& B. G. Andersson (San Francisco, CA: ASP), 194

Rohrmann, R. D., Serenelli, A. M., Althaus, L. G., \& Benvenuto, O. G. 2002, MNRAS, 335, 499

Saio, H., \& Jeffery, C. S. 2000, MNRAS, 313, 671

Schönberner, D. 1979, A\&A, 79, 108

Segretain, L., Chabrier, G., Hernanz, M., Garcia-Berro, E., Isern, J., \& Mochkovitch, R. 1994, ApJ, 434, 641

Straniero, O., Domínguez, I., Imbriani, G., \& Piersanti, L. 2003, ApJ, 583, 878

Tassoul, M., Fontaine, G., \& Winget, D. E. 1990, ApJS, 72, 335

Torres, S., García-Berro, E., Burkert, A., \& Isern, J. 2002, MNRAS, 336 971

Unglaub, K., \& Bues, I. 1998, A\&A, 338, 75

Unglaub, K., \& Bues, I. 2000, A\&A, 359, 1042

van Hoof, P. A. M., et al. 2007, A\&A, 471, L9

van Horn, H. M. 1968, ApJ, 151, 227

Werner, K. 2001, Ap\&SS, 275, 27

Werner, K., \& Herwig, F. 2006, PASP, 118, 183

Werner, K., Rauch, T., \& Kruk, J. W. 2008a, in ASP Conf. Ser. 391, HydrogenDeficient Stars, ed. K. Werner \& T. Rauch (San Francisco, CA: ASP), 239

Werner, K., Rauch, T., \& Kruk, J. W. 2008b, A\&A, 492, L43

Werner, K., Rauch, T., Napiwotzki, R., Christlieb, N., Reimers, D., \& Karl, C. A. 2004, A\&A, 424, 657

Winget, D. E., Hansen, C. J., Liebert, J., van Horn, H. M., Fontaine, G., Nather, R. E., Kepler, S. O., \& Lamb, D. Q. 1987, ApJ, 315, L77

Winget, D. E., \& Kepler, S. O. 2008, ARA\&A, 46, 157

Winget, D. E., Kepler, S. O., Campos, F., Montgomery, M. H., Girardi, L., Bergeron, P., \& Williams, K. 2009, ApJ, 693, L6

Wood, M. A. 1995, Lecture Notes in Physics, Vol. 443 (Berlin: Springer), 41

York, D. G., et al. 2000, AJ, 120, 1579 